once daily for four weeks can be added for very severe infection. Oral treatment is not routinely recommended for community use. Azithromycin is a new long acting azalide currently under evaluation; initial studies suggest that a one time oral dose may be as effective as topical tetracycline (tenth meeting of the WHO programme advisory group on the prevention of blindness, Geneva 1993). The objectives of chemotherapy are to reduce the intensity of trachomatous inflammation and in the long term to reduce the incidence of trichiasis and visual loss. Currently no effective vaccine against $C$ trachomatis exists.

In communities with a prevalence of trichiasis in women over $1 \%$ a campaign of surgical intervention to correct entropion is indicated. Africa has on average one ophthalmologist per million population, ${ }^{10}$ most being in the capital cities and large towns where trachoma is not a problem. Nevertheless, ophthalmic nurses and other paramedics can be trained to perform trichiasis surgery within the community, safely and with a high rate of success. ${ }^{11}$ The recommended operation is tarsal plate rotation. It is important that surgical services are taken to the communities with a high prevalence of trichiasis if those in greatest need are to receive surgery before severe visual loss and blindness develop.

Identifying communities with blinding or potential blinding trachoma using the WHO grading scheme, followed by a campaign of trichiasis surgery; chemotherapy for children with active disease; and face washing and community sanitation to reduce transmission of infection offer a realistic possibility of controlling blindness from trachoma.

ANDREW R POTTER Ophthalmologist

Centre Hospitalier Departemental,

BP 49, Abomey,

Republic of Benin,

West Africa

1 Sandford Smith J. Eye diseases in hot climates. Bristol: Wright, 1986.

2 Thylefors B, Dawson CR, Jones BR, West SK, Taylor HR. A simple system for the assessment of trachoma and its complications. Bull World Health Organ 1987;65:477-83.

3 Dawson CR, Jones BF, Tarizzo ML. Guide to trachoma control. Geneva: World Health Organisation, 1981 .

4 Taylor HR, West SK, Mmbaga BBO, Katala SJ, Turner V, Lynch M, et al. Hygiene factors and increased risk of trachoma in central Tanzania. Arch Ophthalmol 1989;107:1821-5.

5 Courtright P, Sheppard J, Lane S, Sadek A, Schachter J, Dawson CR. Latrine ownership as a protective factor in inflammatory trachoma in Egypt. Br f Ophthalmol 1991;75:322-5.

West SK, Congdon M, Katala SJ, Mele L. Facial cleanliness and risk of trachoma in families. Arch Ophthalmol 1991;109:855-7.

7 Dawson CR, Juster R, Marx R, Daghfous MT, Ben Djerad A. Limbal disease in trachoma and other ocular chlamydial infections: risk factors for corneal vascularisation. Eye 1989;3:204-9.

8 Stevenson D. Davey and Lightbody's. The control of disease in the tropics. London: HK Lewis, 1987:357-85.

9 Chana HS. Blindness prevention and schools. Community Eye Health 1992;9:9-10.

10 Foster A. Who will operate on Africa's 3 million curably blind people? Lancet 1991;337:1267-9.

11 Bog H, Yorston D, Foster A. Results of community-based eyelid surgery for trichiasis due to trachoma. Br $\mathcal{O}$ Ophthalmol 1993;77:81-3.

\title{
Reflex anoxic seizures
}

\section{May be frightening to parents but are nothing to worry about}

A reflex anoxic seizure is a paroxysmal event triggered by a painful or frightening stimulus which, by vagal stimulation, causes pronounced bradycardia or cardiac arrest and consequent relative cerebral ischaemia, thereby inducing an anoxic "seizure" or "attack". ${ }^{-3}$

A typical attack is always provoked, although the provocation may not be witnessed. The most common trigger is sudden pain or fright, such as that due to trapping a finger in a door or a minor blow to the head, particularly the occiput; other provoking factors include febrile illnesses, ${ }^{4}$ venepuncture, ${ }^{5}$ and an excessively hot or cold bath. The painful experience may be accompanied by a gasp or brief cry, after which the child falls, limply, and appears pale, even deathly white, "as though dead." This phase corresponds to the period of asystole, which is brief, usually lasting less than 15 seconds. The child may then rapidly return to normal with or without crying, although she may sleep.

Frequently, though not invariably, stiffening of the body or opisthotonus, clonic movements, upward deviation of the eyes, and urinary incontinence may follow the initial limp phase. ${ }^{3}$ It is these features that may lead to a misdiagnosis of epilepsy, particularly if the painful or frightening trigger is not witnessed. Stephenson believes that the anoxic seizure may, rarely, induce an epileptic seizure, ${ }^{6}$ although I and other authors have never known this to happen.

Attacks may occur very infrequently or many times a day ${ }^{2}$; this may reflect the extent of the vagal hyperexcitability or the degree of the noxious stimulus, or both. Infants and young children (aged 6 months to 2 years $^{2}$ ) are particularly susceptible, but the condition may occur in adolescents and even adults, and in other family members. ${ }^{2}$ Girls are more commonly affected. The condition is common and probably underdiagnosed; epilepsy and (blue) breath holding attacks are the most common misdiagnoses. Occasionally, sudden pain may induce tonic-clonic seizures in patients with epilepsy, but this should be easy to recognise. The precise number of children who experience these attacks is unknown, given the degree of misdiagnosis (one study has estimated that about $8 / 1000$ preschool children are affected ${ }^{2}$ ). The condition may be as common as epilepsy.

The diagnosis is made on the basis of an accurate history (which may be difficult to obtain if the onset was not witnessed). Even when the entire attack has been seen witnesses frequently recall only the stiffness or abnormal movements, leading to a misdiagnosis of a convulsion or grand mal fit and prescription of antiepileptic drugs. ${ }^{7}$ If the history is incomplete or atypical a written description of further events should be sought from parents or other witnesses to establish the diagnosis. Ocular compression has been suggested as a useful diagnostic and therapeutic manoeuvre in those children in whom the condition is suspected or if families require further reassurance. ${ }^{6}$ Ocular compression stimulates the vagus nerve (the oculocardiac reflex), leading to bradycardia, asystole, and, in the electroencephalogram, high voltage slow activity with or without a period of flattening and a clinical attack (the so-called anoxic seizure, or syncopal attack). ${ }^{26}$

Ocular compression may be distressing for the child and must be undertaken with simultaneous electrocardiographic and electroencephalographic monitoring for obvious reasons. The technique should be avoided in children with a known cardiac arrhythmia or glaucoma and in units with limited experience of it. Some authors consider that ocular compression is unnecessary ${ }^{6}$; they believe that the condition can be diagnosed on the basis of the clinical description, and parental reassurance does not depend on a demonstration of the phenomenon, even in a controlled situation.

The phenomenon is benign; the period of cardiac asystole is brief and children do not die or suffer cardiac or cerebral damage. Limited evidence suggests that resolution of the 
attacks usually occurs by early childhood ${ }^{2}$; occasionally the episodes may persist into early adult life.

The most important aspect of treatment is to explain the condition to parents and to reassure them that it is not epilepsy and that the prognosis is good. Atropine has been advocated to prevent the vagal hypersensitivity in those children with frequent, persistent attacks or when parents remain concerned. ${ }^{39}$ Although atropine may be effective, ${ }^{9}$ its pharmacodynamic properties indicate that dosing may need to be frequent, which increases the risk of overdose. Drug treatment has been unnecessary in my and at least one other author's experience. ${ }^{10}$

"Reflex anoxic seizure" is not universally accepted as the most appropriate term for this phenomenon ${ }^{112}$ because the word seizure in many medical (and non-medical) minds is synonymous with epilepsy. ${ }^{13}$ As the condition is often misdiagnosed as epilepsy (this accounted for $15 \%$ of misdiagnoses in one series ${ }^{7}$ ) the description of the attacks as seizures should perhaps be avoided to prevent any additional confusion or concern. ${ }^{13}$ Parents often remember only the word seizure and not what it means, no matter how careful the explanation. ${ }^{1314}$ Because of this, and the suggested mechanism, these attacks have also been called white or type 2 breath holding attacks ${ }^{10}$ (in contrast to the blue or type 1 breath holding attacks), although the primary event is not one of breath holding. Other names include reflex anoxic cerebral seizures with white syncope, ${ }^{3}$ which is too verbose a label, and pallid infantile syncope ${ }^{2}$ or pallid syncopal attacks, which is probably the most popular alternative. ${ }^{113}$ Pallor, however, is not a constant feature..$^{12}$

An awareness of the condition and an accurate history are crucial to both diagnosis and "treatment." This may help to resolve some of the concern that has been expressed recently in the national press ${ }^{15}$ and that has prompted the inception of a self help group for "affected" families.

RICHARD E APPLETON Consultant paediatric neurologist

The Roald Dahl EEG Unit,

Royal Liverpool Children's NHS Trust (Alder Hey),

Liverpool L12 2AP

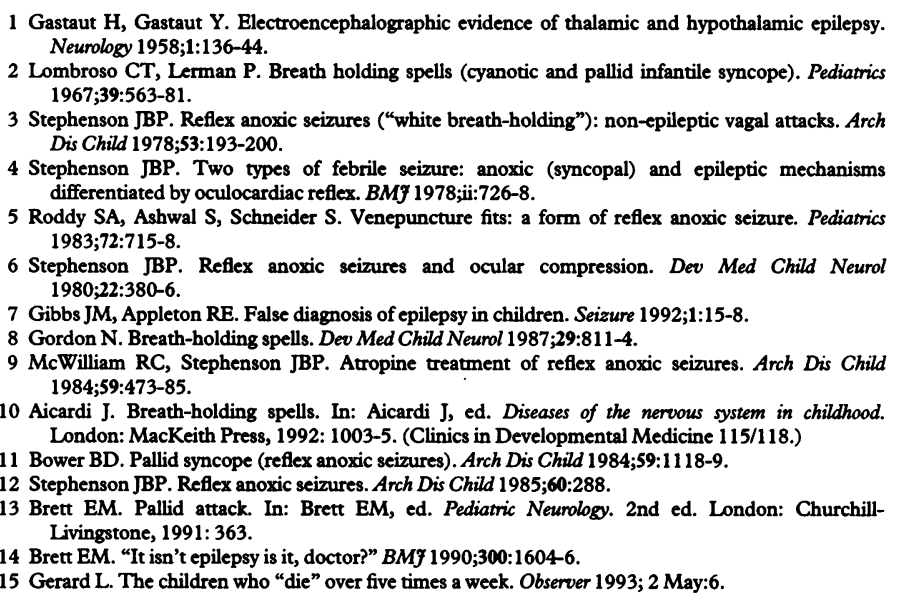

\section{Who needs dual chamber pacing?}

\section{The British guidelines probably exaggerate the needs; proper studies should be done}

Traditional cardiac pacing transformed the lives of a generation of patients with heart block. Victims of Stokes Adams attacks regained their confidence and their driving licences. Those with fatigue and heart failure due to bradycardia were improved. Their pacing systems were simple: a single transvenous electrode positioned in the right ventricle, which was attached to a generator implanted subcutaneously. It paced the heart at 70 beats per minute and was inhibited if the patient's own heart rate exceeded that speed.

These patients fared well. But experience over the past 15 years suggests that they might have fared even better with a more complex system. The addition of an atrial electrode to sense or pace the atria (or do both), linked to a dual chamber pacemaker that then triggers ventricular depolarisation, has many theoretical and practical advantages. Firstly, restoring atrial transport improves stroke volume; secondly, heart rate can respond to effort and further improve exercise capacity ${ }^{1}$ provided that sinus node function is normal; thirdly, compared with single chamber systems it may reduce the risk of the subsequent development of atrial fibrillation ${ }^{2}$ and systemic embolism ${ }^{3}$; fourthly, the pacemaker syndrome-a vague collection of symptoms due to the atria and ventricles contracting asynchronously-is abolished ${ }^{4}$; and lastly, dual chamber pacing is now reliable and effective over years. ${ }^{5}$

The only definite contraindication to dual chamber pacing is established atrial fibrillation, but even then a heart rate response to exercise can be achieved in those with a bradycardia by implanting a generator that senses a bodily changefor example, movement - and accelerates accordingly. Dual chamber and rate response devices are but two of the more spectacular developments in pacing technology. These and others, such as the ability to reprogramme the generator after implantation, have led to such an array of alternatives that in 1991 two groups of experts published guidelines for pacemaker implants, taking into account both the technical advances and the widening indications for this form of treatment. Both the British ${ }^{6}$ and North American ${ }^{7}$ reports recommend the greater use of more complex pacemakers; the document from the British Pacing and Electrophysiology Group contains the suggestion that "the atrium should be paced/sensed unless contra-indicated." This has financial consequences, as the authors predicted.

A dual chamber pacing system costs almost three times as much as a simple ventricular system-about $£ 2100$ compared with $£ 750$ (1991 prices). Wholesale implementation of the British recommendations would increase pacing budgets by an estimated $75 \%$ to $94 \%,{ }^{9}$ although this has been challenged..$^{10}$ The expense does not stop there, however: dual chamber systems are more difficult to insert; follow up appointments take longer; and pacemaker malfunction is more difficult to detect, placing greater demands on technical staff. In addition, the generators will probably not last as long.

The working party of the British Pacing and Electrophysiology Group and others ${ }^{11}$ have argued that costs should not inhibit the promulgation of an ideal pacing practice. Quite so, but a more serious objection is that all the guidelines ${ }^{6711}$ ignore patients and their clinical needs; the recommendations deal solely with conduction systems and their defects.

The average age of the 700 patients who received new pacemaker implants at this hospital in 1990-1 was 76 years; $82 \%$ of the patients from St George's Hospital, London, were over $65 .^{8}$ Most pacemaker patients are elderly. Their exercise 